\title{
LOW-COST OPTICAL CAMERA SYSTEM FOR DISASTER MONITORING
}

\author{
F. Kurz, O. Meynberg, D. Rosenbaum, S. Türmer, P. Reinartz, M. Schroeder \\ German Aerospace Center, 82234 Wessling, Germany - (franz.kurz, oliver.meynberg, dominik.rosenbaum, \\ sebastian.tuermer, peter.reinartz, manfred.schroeder)@dlr.de
}

Commission VIII, WG 1

KEY WORDS: Hazards, Aerial optical camera, Real-time, Performance, Thematic processing, Cost

\begin{abstract}
:
Real-time monitoring of natural disasters, mass events, and large accidents with airborne optical sensors is an ongoing topic in research and development. Airborne monitoring is used as a complemental data source with the advantage of flexible data acquisition and higher spatial resolution compared to optical satellite data. In cases of disasters or mass events, optical high resolution image data received directly after acquisition are highly welcomed by security related organizations like police and rescue forces. Low-cost optical camera systems are suitable for real-time applications as the accuracy requirements can be lowered in return for faster processing times. In this paper, the performance of low-cost camera systems for real-time mapping applications is exemplarily evaluated based on already existing sensor systems operated at German Aerospace Center (DLR). Focus lies next to the geometrical and radiometric performance on the real time processing chain which includes image processors, thematic processors for automatic traffic extraction and automatic person tracking, data downlink to the ground station, and further processing and distribution on the ground. Finally, a concept for a national airborne rapid mapping service based on the low-cost hardware is proposed.
\end{abstract}

\section{INTRODUCTION}

With the rise of new airborne platforms in particular of UAVs there is an increasing demand for low-cost, low-weight and small optical camera systems. These aspects become even more important as the payload of these flying platforms is limited and end users such as police and rescue forces want to equip their proprietary flight squadrons at limited costs.

Also, the possibility of real-time processing of airborne optical camera images in combination with high frame rates paves the way for innovative applications. It is possible to monitor highly dynamic processes like traffic (Rosenbaum, 2008, Leitloff, 2010) or persons (Sirmacek, 2011). DSMs (Digital Surface Models) generated in real time (Zhu, 2010) and real-time orthophoto maps are a valuable data source in different scenarios.

Thus, combining the new airborne platforms and real-time processing capabilities, new applications in the context of disaster monitoring are emerging.

There are three low-cost, real-time optical sensor units operated at DLR, the $3 \mathrm{~K}$ and $3 \mathrm{~K}+$ camera system licensed for the DLR airplanes Cessna and Do228 as well as a sensor unit called CHICAGO integrated in a motorized DLR glider powered by a hydrogen-oxygen fuel cell (Coppinger, 2010). For all sensors, the real-time processing chain is installed aboard the aircraft, i.e. data can be processed directly after the acquisition and sent down to a ground station. A real-time georeferencing processor is implemented followed by thematic processors for automatic traffic detection and automatic person tracking. All hardware components are relatively cheap, except for the GPS/Inertial system from IGI (IGI, 2011). Thus, efforts are made to replace the IMU by a software solution e.g. by optical navigation (Kozempel, 2009), but in the proposed processing chain the GPS/IMU remains included to allow real-time processing. In chapter 2, a short overview over the hardware and software system is given, followed by the evaluation of the system performance in chapter 3 in terms of processing time and quality parameters of the processors.

Chapter 4 describes the concept as well as the investment costs and operational costs for an airborne German wide rapid mapping service. Finally, the pros and cons of the proposed airborne monitoring service are discussed in the context of natural disasters.

\section{SYSTEM OVERVIEW}

\subsection{Hardware}

The system components used for the real time processing chain from the airplane to the ground station are described in (Kurz, 2012). In the following a short summary is given. Each of the $3 \mathrm{~K} / 3 \mathrm{~K}+/ \mathrm{CHICAGO}$ systems consists of three non-metric Canon cameras (Fig. 1). For the 3K system the Canon EOS 1Ds Mark II camera with Canon lenses is used, whereas the successor models 3K+/CHICAGO use the CANON EOS 1Ds Mark III camera with Zeiss lenses. The nominal focal length for $3 \mathrm{~K} / 3 \mathrm{~K}+$ is $50 \mathrm{~mm}$ and for the CHICAGO system $35 \mathrm{~mm}$ in the side-look and $50 \mathrm{~mm}$ in forward / backward direction. The $3 \mathrm{~K}$ and $3 \mathrm{~K}+$ systems are mounted on a ZEISS aerial shock mount ready for the DLR airplanes. The main differences between $3 \mathrm{~K}$ and $3 \mathrm{~K}+/ \mathrm{CHICAGO}$ are the cameras and lenses, the rest of the software components remain the same. The Mark III camera delivers 21.0 MPix compared to 16.7MPix of the Mark II camera. Thus, the ground sample distance (GSD) of an image taken from $1000 \mathrm{~m}$ above ground level (AGL) in nadir direction is $15 \mathrm{~cm}$ and $13 \mathrm{~cm}$ for the $3 \mathrm{~K}$ and the $3 \mathrm{~K}+$ systems, respectively.

The on-board system consists of the optical sensors, the GPS/Inertial system, the processing units, and a C-band microwave data link with a downlink capacity of up to 54 $\mathrm{MBit} / \mathrm{s}$ depending on the distance and bandwidth (Figure 2). 


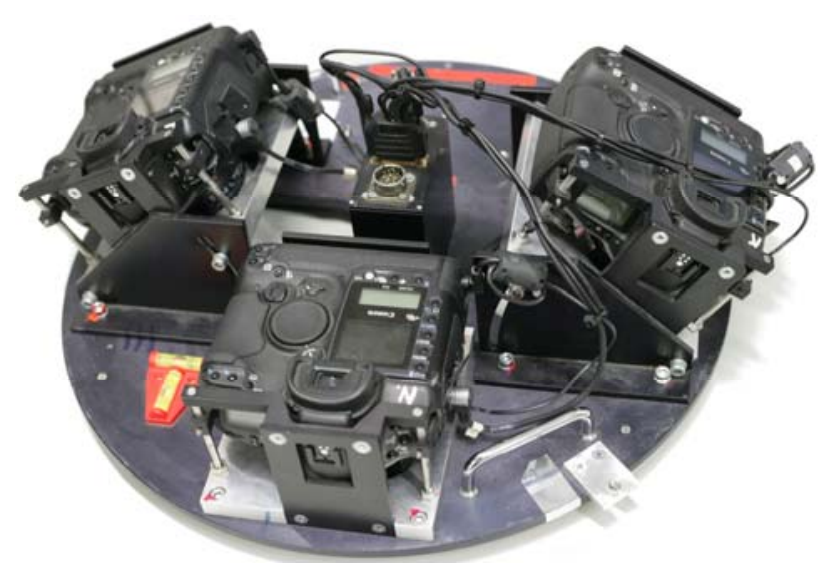

Figure 1 . The $3 \mathrm{~K}+$ camera system

The data from the GPS/Inertial system are used for direct georeferencing of the images. Upon receiving the pre-processed data from the airplane, the mobile ground station processes the data and provides them to the end users via web-based portals (Kurz, 2011).

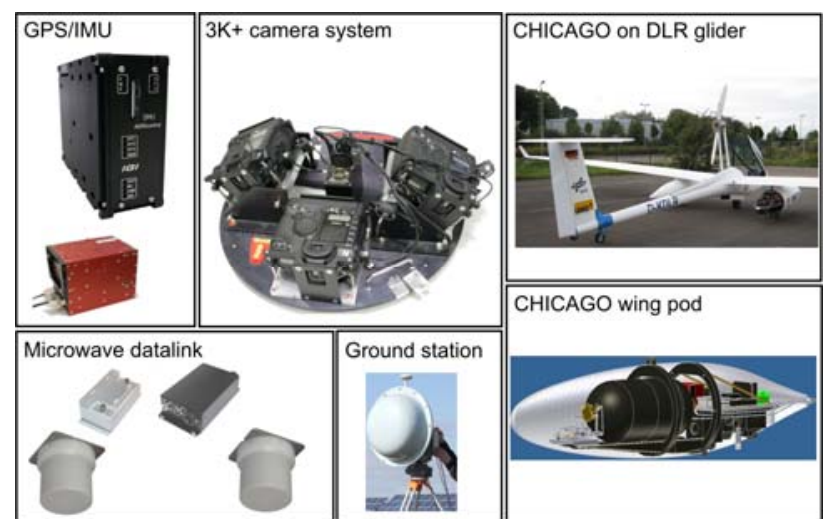

Figure 2. Airborne hardware components and data flow of the $3 \mathrm{~K}$ camera system for the real time processing chain

\subsection{Onboard processing}

The software running on the onboard computers must be capable to process the incoming images in a way that the produced data received on the ground is still up to date and of use for the rescue forces. Moreover large data pile-ups caused by a slow onboard processing module can stall the processing system and must be avoided. These problems are quite likely to happen because the detection and tracking of vehicles or persons need high-resolution images in rapid sequence leading to large amounts of data inside the processing chain.

Therefore, each camera has one dedicated computer for processing the images. Before the actual detection of humans or vehicles starts each image is pre-processed in two major steps. Firstly, after the image is downloaded from the camera the IGI system sends an event date with the exact time stamp, location, and orientation of when the image has been taken to the computer. The synchronization is done with the help of the camera's external flash connector. Secondly, georeferencing and orthorectification take place. The interior and exterior camera parameters, determined by in-flight calibration (Kurz, 2012), and an SRTM DEM are loaded before take-off. After determining the image bounding box the processor calculates the intersection of each image ray with the sensor plane on the graphics processing unit (GPU) rather than on the host's CPU. The program works with NVIDIA's CUDA software library and uses its special memory areas to accelerate the orthorectification. As each pixel can be orthorectified independently this calculation is well-suited for GPU architectures. Only by leveraging the image-processing capabilities of the video card's GPU it is possible to provide high-resolution orthorectified images to the thematic processors on time.

One of the thematic processors extracts fully automatically road traffic data from orthorectified images during the course of a flight. This processing module consists of a vehicle detector and a tracking algorithm. Images are acquired for traffic processing in a so called burst mode. It consists of brief image sequences of few images (3-5 images per burst) with a high repetition rate (up to 3 fps). Every 5-7 seconds a burst is triggered, depending on flight height and flight speed, so that there is nearly no overlap between images of different bursts. This reduces the amount of image data produced in comparison to a continuous recording mode at high frame rate significantly. With this technique we are able to perform automatic traffic data extraction in real-time. To each first image of the burst, road axes from a Navteq road database are overlaid, and vehicles are detected along these roads. Vehicle detection is done by machine learning algorithms AdaBoost and support vector machine, which had been trained intensively on the detection of cars offline prior to flight (Leitloff, 2010). Vehicle tracking is performed between consecutive image pairs within an image burst, based on the vehicle detection in the first image. In the first burst image a template is produced for each detected vehicle and these templates are searched for in the consecutive images by template matching (Rosenbaum, 2010).

\section{SYSTEM PERFORMANCE}

In the following the quality and performance of the onboard processing chain is evaluated. At first the quality of the produced data is discussed and then the real-time performance of the system.

\subsection{Quality of Service}

Products like ortho mosaics and traffic parameters should be generated with sufficient geometric accuracy; $3 \mathrm{~m}$ absolute horizontal position accuracy is assumed as sufficient in particular for the import into GIS or road databases. Table 1 lists the horizontal and vertical georeferencing accuracy separated for the post processing and real time case. For the latter, the images are orthorectified based only on GPS/Inertial system data and the global 25m-resolution SRTM DEM.

\begin{tabular}{|c|c|c|c|}
\hline & \multicolumn{2}{|c|}{$\begin{array}{l}\text { Post processing / } \\
\text { Bundle adjustment }\end{array}$} & \multirow{2}{*}{$\begin{array}{l}\text { Direct georeferencing: } \\
\text { RMSE }^{*}\end{array}$} \\
\hline & $\sigma_{\text {theor }}$ & $\mathrm{RMSE}_{\text {empir }}$ & \\
\hline $\mathrm{X}$ & $0.083 \mathrm{~m}$ & $0.138 \mathrm{~m}$ & $<3 \mathrm{~m}$ \\
\hline $\mathrm{Y}$ & $0.078 \mathrm{~m}$ & $0.365 \mathrm{~m}$ & $<3 \mathrm{~m}$ \\
\hline $\mathrm{Z}$ & $0.400 \mathrm{~m}$ & $0.452 \mathrm{~m}$ & n.a. \\
\hline
\end{tabular}

"Without DEM error, assuming GPS position error $<0.1 \mathrm{~m}$, angle error of inertial system $<0.05^{\circ}$, flight height $1000 \mathrm{~m}$ AGL

Table 1. Georeferencing accuracy of $3 \mathrm{~K}+$ camera system given a bundle adjustment (left). It is only based on GPS/Inertial system measurements (right). 
Figure 2 shows results of the vehicle detection and tracking algorithm. Detected and tracked vehicles are marked by arrows showing the direction of travel with its color representing the vehicle velocity. The correctness of the traffic data obtained from that scene was $95 \%$, the completeness was $85 \%$. This results in a total quality of $81 \%$ which is defined as

$$
\text { Quality }=\frac{\text { truepositives }}{\text { truepositives }+ \text { falsepositives }+ \text { falsenegatives }} \text {. }
$$

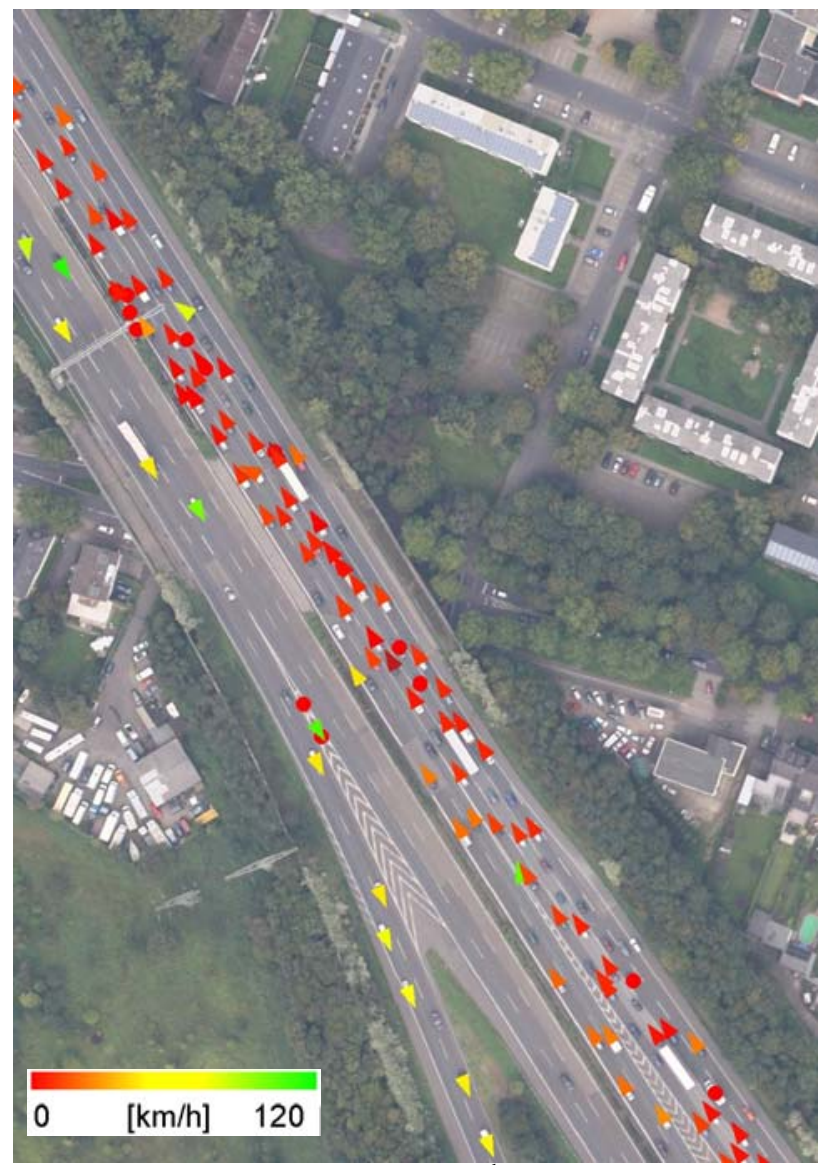

Figure 2. $3 \mathrm{~K}+$ scene obtained on $17^{\text {th }}$ September 2011 close to Cologne/Germany at a flight height of 1500 m AGL.

\subsection{Real-time performance}

During the first hours after arriving in an affected area rescue forces often only need distances or spatial dimensions of buildings or bridges to start working, so up-to-date orthorectified images might be all they need in the beginning. Therefore, it is interesting to know how fast the onboard system is able to provide rescue forces with these images.

The image acquisition and the synchronization with the IGI system hardly take any time compared to the succeeding onboard processing modules and are neglected in the following. As stated earlier, the $3 \mathrm{~K}+$ system can be installed across and along flight track, respectively. This test uses the across track setup (because of the larger coverage) in order to show orthorectified land area as a function of processing time. It mainly depends on the flight height and the changing GSD.

Table 2 lists typical flight heights and the resulting swath width. The $3 \mathrm{~K}+$ system can cover a swath of $1280 \mathrm{~m}$ at 500m AGL and can orthorectify $20 \mathrm{~km}^{2}$ in 3.5 minutes (Fig. 3) with a GSD of $6.5 \mathrm{~cm}$.

\begin{tabular}{|l|l|}
\hline & $3 \mathbf{3}+$ camera system \\
\hline Viewing directions & $1 \times$ nadir, $2 \times \pm 32^{\circ} /$ variable \\
\hline FOV & $\pm 52^{\circ}$ across \\
\hline Coverage / GSD @ 500m & $1280 \mathrm{~m} \times 240 \mathrm{~m} / 6.5 \mathrm{~cm}$ nadir \\
\hline Coverage / GSD @ 1000m & $2560 \mathrm{~m} \times 480 \mathrm{~m} / 13 \mathrm{~cm}$ nadir \\
\hline Coverage / GSD @ 3000m & $7680 \mathrm{~m} \times 1440 \mathrm{~m} / 39 \mathrm{~cm}$ nadir \\
\hline
\end{tabular}

Table 2. Coverage and GSD of the $3 \mathrm{~K}+$ camera system

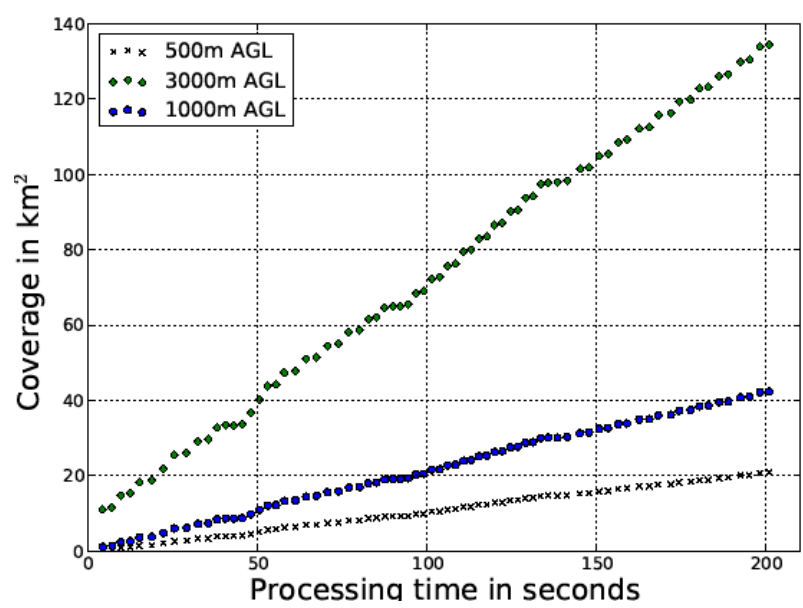

Figure 3. Coverage of onboard processed orthophotos as a function of processing time during a flight.

If the operators at the ground station are more interested in a larger overview of the scene the system can cover a swath of almost $8 \mathrm{~km}$ if it climbs to 3000m AGL. 39cm-resolution images of almost $140 \mathrm{~km}^{2}$ can be sent to the ground after the same processing time of 3.5 minutes (210sec in Fig.3). An important result is the almost linear progress of the coverage at all considered flight levels. If it were a logarithmic progress it would mean that the image processing time cannot keep up with the cruising speed of the airplane, which is typically at 136 knots due to the shutter speed of the cameras. In addition there are almost always longer pauses between single flight strips because of heading for other areas or limitations by flight control. An example of in-flight generated images is shown in Figure 4. In this case, the images cover an area of $35 \mathrm{~km}^{2}$.

A good trade-off between coverage and resolution is flight heights between $1000 \mathrm{~m}$ and $1500 \mathrm{~m}$ AGL because in this case the GSD is small enough to get good results in further object detection algorithms like the traffic processing module. The already mentioned scene in Figure 2 was processed as part of a larger image. When flying in traffic detection mode the time between the bursts is used by the traffic processor to process the last burst. With the current version (described in Section 2.2) it is possible to complete the vehicle detection and tracking before the next image burst is taken. After compressing the results the system sends them directly to the ground with an average data rate of $7 \mathrm{Mbit} / \mathrm{s}$ which is high enough to send all processed data. These results show that the whole onboard processing system is able to operate in real time. 


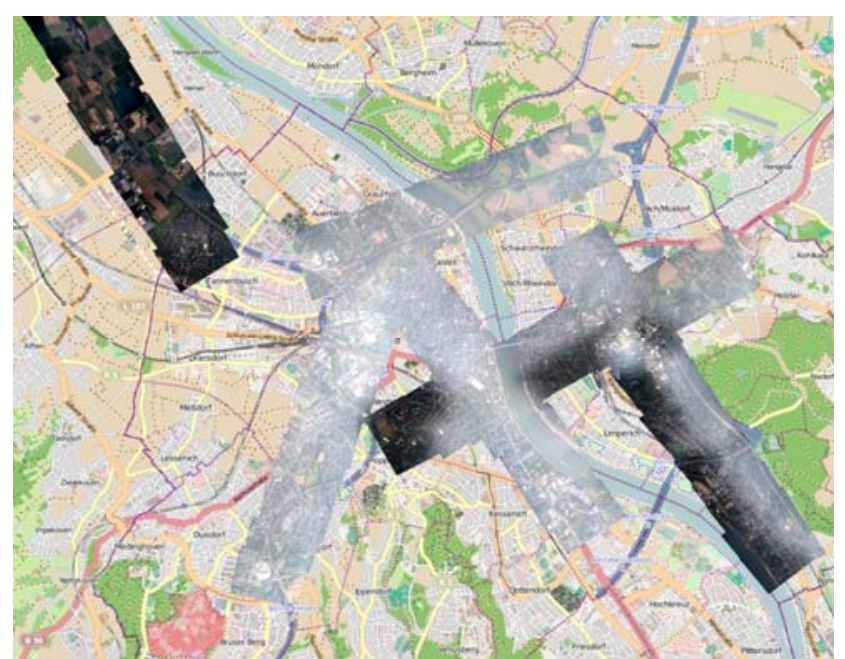

Figure 4. Flight strips at $3^{\text {th }}$ October at main traffic routes in Bonn/Germany. Some parts of the surface were covered by haze.

\section{CONCEPT OF OPERATIONALIZATION}

In this chapter, a concept for an operational airborne rapid mapping service is described. The concept will list the costs for equipment investment and operational costs.

\subsection{Investment costs}

First, the investments in equipment for airborne remote sensing depend on the expected time delay between image acquisition and map delivery. For this, there are different stages of extension for the operational use of this service, starting from traditional airborne acquisitions without onboard processing and online delivery up to the final extension including the real time processing chain.

The different scenarios of airborne and ground based equipment including the hardware costs and the expected reaction times are listed in table 3.

\begin{tabular}{|ll|c|c|c|}
\hline & Scenario & Costs $^{1)}$ & Time $^{2)}$ & Remark \\
\hline A & $\begin{array}{l}\text { No data link, post- } \\
\text { processing }\end{array}$ & $90 \mathrm{k} €$ & $>1 \mathrm{~d}$ & \\
\hline B & $\begin{array}{l}\text { No data link, } \\
\text { onboard processing }\end{array}$ & $120 \mathrm{k} €$ & $<4 \mathrm{~h}$ & $\begin{array}{c}\text { Data are sent } \\
\text { directly after } \\
\text { landing }\end{array}$ \\
\hline C & $\begin{array}{l}\text { Data link, onboard } \\
\text { processing, mobile } \\
\text { ground station (GS) }\end{array}$ & $185 \mathrm{k} €$ & $<6 \mathrm{~h}$ & $\begin{array}{c}\text { Similar to B, } \\
\text { but mob. GS } \\
\text { must be } \\
\text { transferred }\end{array}$ \\
\hline D & $\begin{array}{l}\text { Data link, onboard } \\
\text { processing, net of } \\
\text { stationary antennas }\end{array}$ & $815 \mathrm{k} €$ & $<2 \mathrm{~h}$ & \\
\hline
\end{tabular}

${ }^{1)}$ Only hardware, without airplane, software, licenses, certifications, flight clearances, other infrastructure, etc.

${ }^{2)}$ Envisaged time span between notification and data delivery

Table 3. Comparison of costs and time span for different scenarios

Scenario $\mathrm{A}$ is the cheapest and is basically the traditional airborne acquisition using standard software for orthorectification on the ground station after landing. The processing starts after arrival of the airplane at the ground station. At scenarios B to D, the orthorectification process will be performed onboard using GPS/IMU data, which will drastically reduce the processing time. At the scenarios $C$ and $\mathrm{D}$, the data will be sent directly to ground station. The difference is that in scenario $\mathrm{C}$ the mobile ground station must be transferred to the destination area either in the airplane or by car whereas in scenario D the processed data are directly sent from the airplane to a net of stationary ground antennas. In the latter case, a net of nine receiving and processing stations well distributed over Germany makes direct downlink of data from the airplane possible without additional transfer times of the mobile ground stations (Fig. 5). In this case, a maximum data link range of $100 \mathrm{~km}$ line-of-sight is assumed.

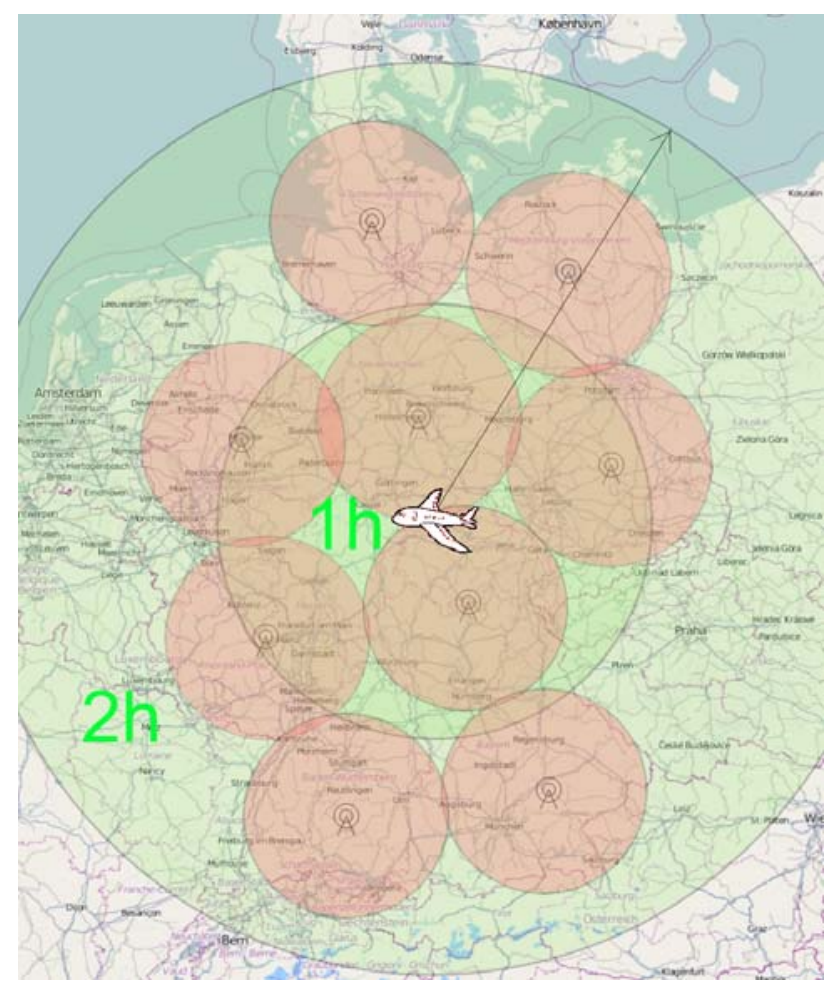

Figure 5. Scenario for a German wide airborne rapid mapping service based on nine stationary receiving stations and one airplane (Scenario D).

\subsection{Operational costs}

The costs for the airplane, the airplane operation, the crews, the processing costs, map production costs, etc. related to the airborne platform are assumed to be mainly independent of the scenarios listed in Table 3. In the following, the costs based on an example are listed in Table 4 to make the costs comparable with satellite based acquisition. The calculations are based on the prices (by end of 2011) at the German Aerospace Center, which are given to external users. In the costs included are the amortisation of the airplane, airplane maintenance and other operational costs assuming 200 days of operation per year. Further, we assume Oberpfaffenhofen close to Munich as home station of the airplane, a $30 \mathrm{~km} \times 30 \mathrm{~km}$ area in Hamburg as target area, and three days of image acquisitions. Further, costs for airborne and ground station crews will be listed which results in altogether $24 \mathrm{k} €$ for the given example. 


\section{REFERENCES}

\begin{tabular}{|c|c|c|}
\hline & Costs & Remark \\
\hline Aircraft & $18 \mathrm{k} €$ & $\begin{array}{c}12 \text { flight hours, 3 days, } \\
\text { includes amortisation }\end{array}$ \\
\hline Fees & $3 \mathrm{k} €$ & $\begin{array}{c}\text { Flight clearance, airport } \\
\text { fees, etc. }\end{array}$ \\
\hline $\begin{array}{c}\text { Personnel } \\
\text { costs }\end{array}$ & $3 \mathrm{k} €$ & $\begin{array}{c}\text { Travel costs, wages, } \\
\text { etc. }\end{array}$ \\
\hline & $24 \mathrm{k} €$ & \\
\hline
\end{tabular}

Table 4. Costs for airborne monitoring of three coverages in Hamburg including crews and operators.

\subsection{Price calculation}

The provider of an airborne rapid mapping service acquires high resolution georeferenced image data in real time. The service can be activated by international or national organizations, e.g. by the International Charter, and should therefore be comparable to commercial high resolution satellite scenes in terms of costs, delivery times, etc.

Based on the example Hamburg, three satellite scenes with highest resolution e.g. Worldview, Quickbird with high priority cost around 20 to $30 \mathrm{k} €$, i.e. for this example the operational costs are comparable. More generalized, the airborne operational costs for providing georeferenced image scenes are approximately $10 € / \mathrm{km}^{2}$. For economic feasibility, the final prices for airborne image scenes will be higher to cover the investment costs (Tab. 4). Thus, the final price will depend mainly on the desired reaction time and the targeted regions in terms of the different scenarios A to D.

\section{CONCLUSIONS}

Different low-cost camera systems for real-time disaster monitoring are presented and the major differences between them are clarified. The $3 \mathrm{~K}$ and $3 \mathrm{~K}+$ camera system mounted on a turboprop-engined aircraft enables the police and other rescue forces to have a detailed and up-to-date overview of disaster areas. The CHICAGO camera system has slightly less coverage but is able to monitor events and other hot spots for a longer period of time. The processing system, which is closely connected to the cameras aboard the aircraft, has the advantage of having direct access to the uncompressed and fully-detailed images. These large images are handled efficiently with the help of GPU-accelerated processing and modern image processing algorithms. The orthorectification and the trafficdata extraction are fast enough to allow a continuous image acquisition with a high quality index. Depending on the mission goals high-resolution orthorectified images and/or current traffic data can be sent to the ground in real time. Possible operational scenarios are discussed and differ mainly in the costs depending on the desired reaction time. The assets and drawbacks of operational airborne emergency mapping are discussed in comparison to satellite image acquisition.

In the future, it is planned to design a highly integrated, lightweight sensor in order to equip other smaller aircrafts with similar monitoring systems. Moreover, other object recognition methods are going to be implemented to extend the system's field of applications, e.g. crowd analysis.
Coppinger, R., 2010. Fuel cell motor-glider basis for endurance UAV. In: Flight International 177 (5233): 25

IGI 2011. Ingenieur Gesellschaft für Interfaces mbH, http://www.igi.eu (22.12.2011)

Kozempel, K., \& Reulke, R., 2009. Camera Orientation Based on Matching Road Networks. In: Image and Vision Computing New Zealand, IVCNZ '09. 24th International Conference, 237 242,

Kurz, F., 2009. Accuracy assessment of the DLR 3K camera system. In: DGPF Tagungsband, 18. Jahrestagung 2009.

Kurz, F., Rosenbaum, D., Leitloff, J., Meynberg, O. \& Reinartz, P., 2011. Real-time camera system for disaster and traffic monitoring. In: Proceedings of International Conference on SMPR 2011. International Conference on Sensors and Models in Photogrammetry and Remote Sensing, 18.-19. Mai 2011, Teheran, Iran.

Kurz, F., Türmer, S., Meynberg, O., Rosenbaum, D., Runge, H., Reinartz, P. 2012. Low-cost camera system for real-time applications. In: PFG 2012/2. pp. 157-176.

Leitloff, J., Hinz, S. \& Stilla, U., 2010. Vehicle Detection in Very High Resolution Satellite Images of City Areas. In: IEEE Transactions on Geoscience and Remote Sensing 48 (7): 27952806.

Rosenbaum, D., Kurz, F., Thomas, U., Suri, S. \& Reinartz, P., 2008. Towards automatic near real-time traffic monitoring with an airborne wide angle camera system. In: European Transport Research Review, 1(1):11-12.

Rosenbaum, D, Leitloff, J., Kurz, F., Meynberg, O. \& Reize, T., 2010. Real-Time Image Processing for Road Traffic Data Extraction from Aerial Images. In: Technical Commission VII Symposium 2010 - June 2010, Vienna, Austria

Sirmacek, B. \& Reinartz, P., 2011. Automatic crowd density and motion analysis in airborne image sequences based on a probabilistic framework. In: Proceedings of the 2nd IEEE ICCV Workshop on Analysis and Retrieval and Tracked Events and Motion in Imagery Streams (ARTEMIS'11), Nov. 2011, Barcelona, Spain.

Zhu, K., d'Angelo, P. \& Butenuth, M., 2010: Comparison of Dense Stereo using CUDA. In: $11^{\text {th }}$ European Conference on Computer Vision (ECCV) - Crete, Greece. 